\title{
Effect of NEFA and Glucose Levels on CPT-I mRNA Expression and Translation in Cultured Bovine Hepatocytes
}

\author{
Chuang XU' ${ }^{1)}$, Zhe WANG ${ }^{2}$, Ri-he ZHANG ${ }^{2}$, Hong-you ZHANG ${ }^{1)}$, Shi-xin FU ${ }^{1)}$ and Cheng XIA ${ }^{1) *}$ \\ ${ }^{1)}$ Department of Clinical Veterinary Medicine, Animal Science and Technology College, Heilongjiang Bayi Agricultural University, \\ Daqing, 163319 and ${ }^{2}$ Department of Clinical Veterinary Medicine, Animal and Veterinary College, Jilin University, Changchun, Jilin \\ Province, 130062, PR China
}

(Received 19 April 2010/Accepted 29 July 2010/Published online in J-STAGE 12 August 2010)

\begin{abstract}
The objective of this study was to determine the effects of NEFA and glucose on carnitine palmitoyltransferase-I (CPT-I) mRNA expression in cultured bovine hepatocytes using real-time reverse transcription polymerase chain reaction and ELISA methods. The results indicated that CPT-I transcription increased gradually, but that CPT-I translation was not significantly changed, with glucose concentrations ranging from 0 to $3.0 \mathrm{mmol} / \mathrm{L}(P<0.01)$. Furthermore CPT-I transcription and translation were enhanced significantly when the NEFA concentrations increased from 0 to $1.2 \mathrm{mmol} / \mathrm{L}$ and decreased significantly when the NEFA concentrations increased from 1.2 to $4.8 \mathrm{mmol} / \mathrm{L}(P<0.01)$. A high concentration NEFA was found to reduce fatty acid oxidation, potentially explaining the development from NEB to ketosis in dairy cows.
\end{abstract}

KEY WORDS: CPT-I, fatty acid transport, glucose, hepatocytes, NEFA.

J. Vet. Med. Sci. 73(1): 97-101, 2011

The main physiological characteristic of peripartum cows is negative energy balance (NEB) [5]. Dairy cows display a relatively low concentration of blood glucose and concurrent high levels of non-esterified fatty acids (NEFA) in the initial stage of NEB [12]. Elevated blood NEFA and low blood glucose are the two key characteristics responsible for the initial serology of NEB [16], and these factors may have an important influence on the development of NEB. Complete oxidation of fatty acids (FAs) in the liver can improve metabolic processes and provide more ATP to relieve NEB and diminish the ketone concentration in blood or triacylglycerol accumulation in the liver. Therefore, the oxidation metabolism pathway of NEFA may be the main pathway providing energy during NEB and concurrent low blood glucose [2, 9]. The transfer of activated FAs (acyl-CoA) into mitochondria is controlled by the enzyme carnitine palmitoyltransferase-I (CPT-I). This step positively correlates with the velocity of transfer of acyl-CoA into mitochondria [6] and controls the process of FA metabolism.

The objective of this study was to detect the effect of NEFA and glucose on CPT-I mRNA expression and translation levels in bovine hepatocytes in vitro. Our findings provide an insight into how the two initial serology factors of NEB, namely a high NEFA concentration and low blood glucose level, affect FA oxidation metabolism.

Hepatocyte cells were cultured as described previously $[7,19]$, with some modifications to optimize the procedures for bovine liver cells. Hepatocytes were isolated from an excised lobe and plated as described previously [15], with some modifications. The cultured cells were cultured in six-

\footnotetext{
* Correspondence to: Xia, C., Department of Clinical Veterinary Medicine, Animal Science and Technology College, Heilongjiang Bayi Agricultural University, Daqing, 163319, PR China.

e-mail: xcwlxyf@sohu.com
}

well plates at a density of $10^{6}$ cells $/ \mathrm{cm}^{2}$ (Costar, Corning Incorporated, Corning, NY, U.S.A.). After a 4-hr attachment period, the media and unattached cells were aspirated and replaced with RPMI 1640 culture medium containing $10 \%$ fetal bovine serum. The cells were then cultured at $37^{\circ} \mathrm{C}$ in $5 \% \mathrm{CO}_{2}$. Every $24 \mathrm{hr}$, the media were aspirated and refreshed [4, 18]. At $72 \mathrm{hr}$, liver cells was cultured with glucose- and NEFA-free RPMI 1640 culture medium. NEFA and glucose were added to the media separately [15]. The $10 \mathrm{mmol} / l$ storage solution of NEFA contained $4.35 \mathrm{mmol} / l$ oleic acid, $0.49 \mathrm{mmol} / l$ linoleic acid, $3.19 \mathrm{mmol} / l$ palmitic acid, $1.44 \mathrm{mmol} / l$ stearic acid and $0.53 \mathrm{mmol} / l$ palmitoleic acid. The final concentrations of NEFA in the media were $0,0.3,0.6,1.2,2.4$ and $4.8 \mathrm{mmol} / l$. The final concentrations of glucose in the media were $0,1.0,1.5,2.0,2.5$ and 3.0 $\mathrm{mmol} / l$. The concentrations of NEFA and glucose used in this study were chosen according to the normal hematology standards of dairy cows during NEB. Each experiment was repeated three times.

After $12 \mathrm{hr}$ of culture, total RNA was extracted from cells according to groupings with a commercially available RNA isolation kit (TriPure, Roche). Total RNA was then treated with RNase-free DNase I (Takara) according to the instruction manual. The RNA concentration was determined by spectrophotometric measurement in $5 \mu l$ capillaries using a GeneQuant II RNA/DNA Calculator (Pharmacia Biotech, Cambridge, UK) [9, 12]. Oligonucleotide primers specific for CPT-I (GenBank accession no. NM_001034349) and $\beta$ actin (GenBank accession no. NM 173979) were designed using the Primer Express ${ }^{\mathrm{TM}}$ Version 2.0 software (PE Applied Biosystems, Inc., Foster, CA, U.S.A.) (Table 1).

Transcription of the CPT-I and $\beta$-actin genes was evaluated on the basis of mRNA copy number $/ l \mu \mathrm{g}$ of total RNA using real-time QRT-PCR with a SYBR Green Quantitect RT-PCR Kit (QIAGEN, Valencia, CA, U.S.A.). The QRT- 
Table 1. Primers used for amplification of the target genes and the internal control genes

\begin{tabular}{llc}
\hline Gene name & Sequence of primers $\left(5^{\prime}-3^{\prime}\right)$ & Length of amplicon (bp) \\
\hline CPT-I & \begin{tabular}{l} 
Forward: GGTCAACAGCAACTACTACG \\
\multirow{3}{*}{-actin }
\end{tabular} & $\begin{array}{l}\text { Reverse: TGAACATCCTCTCCATCTGG } \\
\text { Forward: GTCATCACCATCGGCAATGAG } \\
\text { Reverse: GCTAACAGTCCGCCTAGAAGCA }\end{array}$ \\
\hline
\end{tabular}

PCR assay was performed in triplicate for each sample. The $\beta$-actin gene was also analyzed to monitor RT-PCR efficiencies and to provide an internal reference. Expression of the $\beta$-actin gene was analyzed using specific primers (Table 1) $[8,14]$. To simultaneously detect the expression profile of the genes of interest, we used the $\beta$-actin gene as an internal reference gene at eight different 10 -fold dilutions (from $10^{-2}$ to $\left.10^{-9}\right)$. Amplification plots for each dilution of the control template were used to determine the $\mathrm{Ct}$ value. A standard curve was generated by plotting the $\mathrm{Ct}$ values against the log of the $\beta$-actin cDNA copy number. CPT-I mRNA abundance was displayed as the CPT-I mRNA copy number of the $1 \mu \mathrm{g}$ of total RNA/ $\beta$-actin mRNA copy number. Melting curve analysis was carried out for each run to confirm the specificity of amplification and the absence of primer dimers $[8,14]$.

Cells were harvested from the culture dishes by scraping, recovered by sedimentation and stored at $-80^{\circ} \mathrm{C}$ prior to analysis. Frozen cell pellets, containing about $25 \times 10^{6}$ cells/treatment group (3 dishes), were homogenized in 0.1 $\mathrm{M}$ of potassium phosphate, $\mathrm{pH} 7.4,1 \mathrm{mM}$ EDTA and 20\% glycerol with a Dounce manual homogenizer. Particulate matter was removed by centrifugation for $2 \mathrm{~min}$ at $10,000 \times$ $g$ and stored in aliquots at $-80^{\circ} \mathrm{C}$. Total protein was assayed using Bradford protein assay reagent (Bio-Rad) with bovine serum albumin as the standard. The protein content in the cultured liver cells was determined to calculate the CPT-I protein content per unit of total protein. CPT-I protein levels are presented as the CPT-I protein contents/total protein contents for each sample. Using this method, we corrected for any experimental error caused by differences in the weights of livers [3].

CPT-I was measured using an ADL goat anti-cow carnitine palmitoyltransferase 1 kit (catalogue no. QRCT3013321100845EIA \UTL, Adlitteram Diagnostic Laboratories) according to the manufacturer's instructions. Briefly, a standard sample and liver cell samples were incubated with biotinylated CPT-I in microtitration wells that had been coated with another CPT-I of defined and unique epitope specificity. After incubation and washing, the wells were treated with streptavidin labeled with horseradish peroxidase (HRP). The wells were then washed again, and a TMB substrate solution was added. Color development was proportional to the amount of bound CPT-I. The stop solution changed color from blue to yellow, and the intensity of the color was measured at $450 \mathrm{~nm}$. The minimum detectable concentration of CPT-I was $0.01 \mu \mathrm{g} / \mathrm{ml}$.

Statistical analysis of the data was carried out using the SPSS software with the significance level set at $P<0.05$.
The values were expressed as median values. Friedman's nonparametric ANOVA and Tukey' post hoc test were applied to compare the differences between the expression levels of the genes of interest. Data are expressed as the mean \pm SD. $P<0.05$ was considered significant.

In experiment I, decreased CPT-I mRNA levels were detected in cultured bovine hepatocytes with increasing glucose concentrations in the culture media (Fig. 1). There were significant differences in the CPT-I mRNA levels between the $0 \mathrm{mmol} / l$ group and the $2.0 \mathrm{mmol} / l, 2.5 \mathrm{mmol} / l$ and $3.0 \mathrm{mmol} / l$ groups $(P<0.05)$. Regarding the CPT-I protein content, there were no obvious changes in the CPT-I levels of cultured bovine hepatocytes with increasing glucose concentrations in the culture media $(P>0.05)$. CPT-I expression was not inhibited even when the glucose concentration in the culture media was $0 \mathrm{mmol} / l$. There were no significatn differences in CPT-I transcription and translation levels at the same glucose concentration among the three repeated treatments $(P>0.05)$.

In experiment II, NEFA was found to effect CPT-I transcription and translation either by upregulation or downregulation, depending on the concentration of NEFA in the media (Fig. 2). Increasing CPT-I transcription and translation levels were observed in cultured bovine hepatocytes when the concentration of NEFA was less than $1.2 \mathrm{mmol} / \mathrm{l}$, and decreasing CPT-I transcription and translation levels were observed when the concentration was more than 2.4 $\mathrm{mmol} / l(P<0.01)$. At a NEFA concentration of $4.8 \mathrm{mmol} / \mathrm{l}$, CPT-I was not detected.

FA metabolism differs considerably between monogastric and ruminant species. The regulation of the key enzymes involved may differ accordingly. The CPT-I (EC 2.3.1.21) gene is the key locus for the control of long-chain FA $\beta$-oxidation and liver ketogenesis in dairy cows [13]. Mitochondrial acylcarnitine synthesis is an important control point in the partitioning of cytosolic FAs for mitochondrial $\beta$-oxidation. Only after the first step in this pathway, the transfer of long-chain FAs to mitochondria, is complete can FA oxidation of the energy supply commence.

NEB is a major problem associated with transition dairy cows, which leads to quick blood glucose drawdown and body fat mobilization [16]. Therefore, the blood NEFA concentration is subsequently increased. Low blood glucose levels and high blood NEFA levels are the most important characteristics of NEB. During NEB, when the blood glucose levels are low, the blood glucose is unable to provide enough energy to help relieve NEB [2]. Gluconeogenic substrates such as amino acids (alanine, aspartate and glutamate) and short chain fatty acids (propionate and lac- 


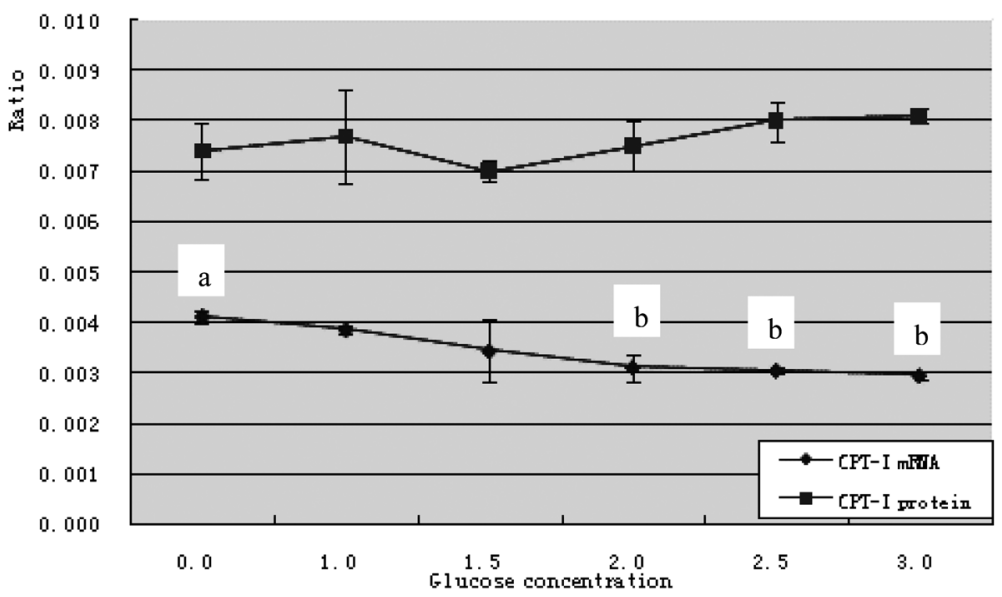

Fig. 1. Effect of different concentrations of glucose on CPT-I transcription and translation. Decreased CPT-I mRNA levels were observed with increasing glucose concentrations in the culture media of bovine hepatocytes. Significant differences in CPT-I mRNA abundance were detected between the $0 \mathrm{mmol} / l$ group and the $2.0 \mathrm{mmol} / l, 2.5 \mathrm{mmol} / l$ and $3.0 \mathrm{mmol} / l$ groups $(P<0.05)$, whereas no significant difference in CPT-I mRNA abundance was detected between any other two groups $(P>0.05)$. There was no obvious change in the CPT-I protein levels with increasing glucose concentrations in the culture media of bovine hepatocytes. Note: Different small letters indicate significant differences, and the same small letters or no letters indicate no significant difference between different concentration sets in the same line. Values are means \pm SD. Different letters, a and b, indicate significant difference between the parameters of CPT-I mRNA or CPT-I protein $(P<0.05)$

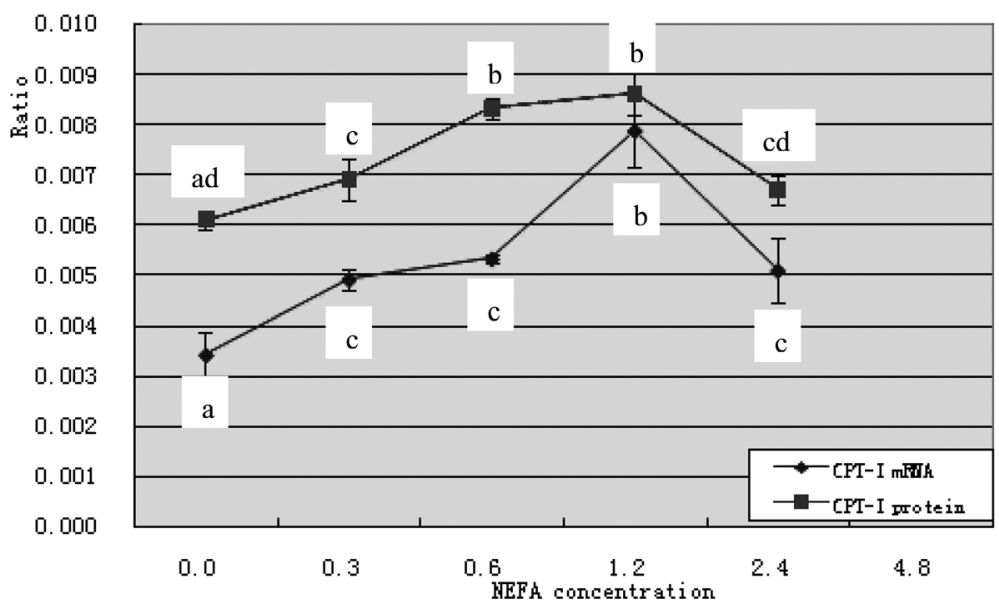

Fig. 2. Effect of different concentrations of NEFA on CPT-I transcription and translation. NEFA affected CPT-I transcription and translation either by upregulation or downregulation depending on the concentration of NEFA in the media. Increasing CPT-I transcription and translation levels in cultured bovine hepatocytes were observed when the concentration of NEFA was less than $1.2 \mathrm{mmol} / l$, and decreasing CPT-I transcription and translation levels were observed when the concentration was more than $2.4 \mathrm{mmol} / l(P<0.01)$. At a concentration of $4.8 \mathrm{mmol} / l$ of NEFA, CPT-I was not detected. Note: Different small letters indicate significant differences, and the same small letters or no letters indicate no significant differences between different concentration sets in the same line. Values are means \pm SD. Different letters, a, b, c and d, indicate significant difference between the parameters of CPT-I mRNA or CPT-I protein $(P<0.05)$.

tate), which could provide an alternative energy source, were also decreased in the cows during NEB. The gluconeogenic rates of all of the substrates mentioned above were significantly decreased during prolonged energy deficit. FAs may therefore be the main energy substrates during this condition [9]. The liver is the most active FA metabolic organ in cows [12]. Therefore, the oxidative metabolism of high levels of blood NEFA in the livers of NEB cows may be important in providing energy for the relief of NEB.
However, the mechanism by which NEFA and glucose, the blood parameters initially altered in NEB cows, affect CPT$\mathrm{I}$, the key enzyme for the control of long-chain FA $\beta$-oxidation, transcription and translation levels in cultured bovine hepatocytes remains unclear. Such findings may help to explain how a single factor, i.e., low blood glucose or high blood NEFA, affects FA oxidation and may provide further insight into understanding the development of NEB.

In our study, no significant changes in CPT-I levels were 
observed with increasing glucose concentrations from 0 $\mathrm{mmol} / \mathrm{l}$ to $3.0 \mathrm{mmol} / \mathrm{l}$ in the culture media of bovine hepatocytes $(P>0.05)$. The normal blood glucose level in cows is about $2.8 \mathrm{mmol} / l$, and the glucose level in the NEB cows was $2.0 \mathrm{mmol} / l$. In the present study, the low level of glucose did not influence the CPT-I mRNA level. While the blood glucose level was increased (more than $2.0 \mathrm{mmol} / \mathrm{l}$ ), CPT-I mRNA expression was decreased and CPT-I enzymes were unaffected, indicating that low glucose control CPT-I activity through influence CPT-I mRNA expression. CPT-I activity was not found to be inhibited at low glucose concentrations, indicating that a low level of glucose did not affect CPT-I translation or FA transfer into mitochondria. This suggested that a single factor characteristic of NEB in cows, i.e., low blood glucose level, did not affect CPT-I activity and FA oxidation. Energy is produced continually from FA oxidation under low blood glucose conditions, and this process is important in slowing down the development of NEB. However, a high NEFA concentration (more than $1.2 \mathrm{mmol} / l$ ) can inhibit CPT-I transcription and translation. Therefore, a high blood NEFA concentration in NEB cows can induce CPT-I activity and energy production by FA oxidation. This result is similar to the findings of Mizutani et al. [11], who found that clinical fatty liver unrelated to calving appears to be associated with a decrease in hepatic CPT activity [11]. However, this contradicts the findings of another study in which hepatic CPT activity showed no correlation with serum phospholipid, free FA, triglyceride or total cholesterol concentrations [10]. CPT-I activity can also be regulated by leptin. Leptin increases FA oxidation by stimulating the activity of CPT-I [17]. The plasma concentrations of leptin correlate with the body mass index. In the initial stage of NEB, dairy cows have a good body mass index, and the concentration of circulating leptin is high, which promotes the activity of CPTI and FA oxidation, providing energy. However, as NEB progresses to a more severe stage, the body mass index decreases, and the blood NEFA concentration increases rapidly. When the blood NEFA concentration reaches sufficiently high level, it inhibits the activity of CPT-I [17]. The position of CPT-I in FA metabolism makes it a primary site for regulation of hepatic ketogenesis, and non-ruminant studies have concluded that the hepatic output of ketone bodies correlates with CPT-I activity [1]. The higher rates of gluconeogenesis and ketogenesis in early lactation are associated with higher hepatic CPT-I activity [1]. Ketogenesis is regulated by CPT-I activity. High CPT-I activity promotes gluconeogenesis, and low CPT-I activity promotes ketogenesis. Taken together with our results, in the early stage of NEB, it is possible that low levels of glucose do not inhibit CPT-I activity, but instead decrease the CPT-I mRNA level, resulting in accelerated gluconeogenesis, and that high levels of NEFA inhibit CPT-I activity during the severe stage of NEB, promoting hepatic ketogenesis and resulting in cow ketosis. Therefore, the high NEFA concentration characteristic of NEB cows affects hepatic CPT-I activity and FA oxidation metabolism and promotes hepatic ketogenesis, which may explain the development of NEB into ketosis.

In our study, a low level of glucose did not affect CPT-I activity in cultured bovine hepatocytes, indicating that FAs can continue to undergo oxidation and supply energy under low glucose conditions, especially in the early stage of low blood glucose. However, CPT-I was significantly inhibited by high NEFA concentrations in cultured bovine hepatocytes, reducing FA oxidation, promoting hepatic ketogenesis and potentially leading to ketosis.

ACKNOWLEDGMENTS. This research was supported by the National Natural Science Foundation of China (30871897), Natural Science Foundation of HLJ (QC2009C32), Department of Education of HLJ (1151G029) and FARM (HNKXIV-08-01-09). Thanks to Master R.H. Zhang of Jilin University for experimental assistance.

\section{REFERENCES}

1. Aiello, R. J., Kenna, T. M. and Herbein, J. H. 1984. Hepatic gluconeogenic and ketogenic interrelationships in the lactating cow. J. Anim. Sci. 67: 1707-1715.

2. Barbara, L.G. 1997. Effects of the monensin controlled-release capsule on ruminal parameters and the occurrence of subclinical ketosis in transition dairy cows. A thesis Presented to The Facuity of Graduate Studies of The University of Guelph.

3. David, J., Waxman, J.J., Sharda, N. and Hugo, J. 1990. Phenobarbital induction of cytochromes P-450. Biochem. J. 271: 113-119.

4. Donkin, S.S. and Armentano, L.E. 1993. Preparation of extended in vitro cultures of bovine hepatocytes that are hormonally responsive. J. Anim. Sci. 71: 2218-2227.

5. Dann, H.M. and Drackley, J.K. 2005. Carnitine palmitoyltransferase I in liver of periparturient dairy cows: effects of prepartum intake, postpartum induction of ketosis, and periparturient disorders. J. Anim. Sci. 88: 3851-3859.

6. Hoppel, C.L. 1982. Carnitine and carnitine palmitoyltransferase in fatty acid oxidation and ketosis. Fed. Proc. 41: 2853 2857.

7. Kramer, K., Markwitan, A. and Pallauf, J. 1993. Studies on the metabolism of metallothionein and alkaline phosphatase of adult rat primary hepatocyte cultures: role off etal calfserum and agonists of the phosphoinositide cascade. Ernahrungs 32: 176-186.

8. Lucia, F., Fabrizio, A., Dario, D.M., Elisabetta, D. and Paolo, A. 2007. SYBR Green Real-Time PCR Method To Detect Clostridiumubotulinum TypeA. Appl. Environ. Microb. 73: 2891-2896.

9. Mills, S.E., Beitz, D.C. and Young, J.W. 1986. Evidence for Impaired Metabolism in Liver During Induced Lactation Ketosis of Dairy Cows. J. Dairy Sci. 69: 362-370.

10. Mizutani, H., Sako, T., Takemura, N., Koyama, H., Yamaguchi, M. and Motoyoshi, S. 1997. Hepatic carnitine palmitoyltransferase activity in cattle. J. Vet. Med. Sci. 59: 1067-1069.

11. Mizutani, H., Sako, T., Toyoda, Y., Kawabata, T., Urumuhang, N., Koyama, H. and Motoyoshi, S. 1999. Preliminary studies on hepatic carnitine palmitoyltransferase in dairy cattle with or without fatty liver. Vet. Res. Commun. 23: 475-480.

12. Melendez, P., Goff, J.P., Risco, C.A., Archbald, L.F., Littell, 
R. and Donovan, G.A. 2006. Incidence of subclinical ketosis in cows supplemented with a monensin controlled-release capsule in Holstein cattle, Florida, USA. Prev. Vet. Med. 73: 33-42.

13. Price, N.T., Jckson, V.N., Leij, F.R., Cameron, J.M., Travers, M.T., Bartelds, B., Huijkman, N.C. and Zammit, V.A. 2003. Cloning and expression of the liver and muscle isoforms of ovine carnitine palmitoyltransferase 1: residues within the Nterminus of the muscle isoform influence the kinetic properties of the enzyme. Biochem. J. 15: 871-879.

14. Strzalka, B., Dorecka, M., Stanik, A., Kowalczyk, M., Kapral, M., Romaniuk, W., Mazurek, U. and Swiatkowska, L. 2008. Quantitative Analysis of Transforming Growth Factor Isoforms mRNA in the Human Corneal Epithelium. Folia Biologica (Praha) 54: 46-52.

15. Xu, C. and Zhe, W. 2006. Effect of propionate, pyruvate and beta -hydroxybutyric acid on pyruvate carboxylase mRNA expression of in vitro culture bovine hepatocytes. Anim. Sci. J. 77: 440-446.

16. Xu, C., Wang, Z., Liu, G.W., Li, X.B., Xie, G.H., Xia, C. and
Zhang, H.Y. 2008. Metabolic characteristic of the liver of dairy cows during ketosis based on comparative proteomics. AsianAust. J. Anim. Sci 21: 1003-1008.

17. Yamagishi, S.I., Edelstein, D., Du, X.L., Kaneda, Y., Guzmán, M. and Brownlee, M. 2001. Leptin induces mitochondrial superoxide production and monocyte chemoattractant protein1 expression in aortic endothelial cells by increasing fatty acid oxidation via protein kinase A. J. Biol. Chem. 276: 25096 25100 .

18. Zhu, L.H., Strang, B.D. and Armentano, L.E. 2000. Effects of triglyceride accumulation on induction of urea synthesis by glucagons and dexamethasone in monolayer cultures of bovine hepatocytes. J. Anim. Sci. 78: 1659-1666.

19. Zavana, B., Bruna, P., Vindignib, V., Amadoric, A., Habelerc, W., Pontissod, P., Montemurroe, D., Abatangeloa, G. and Cortivo, R. 2005. Extracellular matrix-enriched polymeric scaffolds as a substrate for hepatocyte cultures: in vitro and in vivo studies. Biomaterials 26: 7038-7045. 